\title{
Is penicillin prophylaxis effective against bacterial endocarditis?
}

\author{
In people at increased risk of bacterial endocarditis does prophylactic penicillin \\ before invasive dental procedures affect mortality, serious illness or \\ endocarditis incidence?
}

Oliver R, Roberts GJ, Hooper L. Penicillins for the prophylaxis of bacterial endocarditis in dentistry (Cochrane Review). In: The Cochrane Library, Issue 2, 2004. Chichester, UK: John Wiley \& Sons, Ltd.

Data sources Cochrane Oral Health, Heart and Infectious Diseases Groups' Trials Registers Cochrane Central Register of Controlled Trials (CENTRAL) OLDMEDLINE; EMBASE SIGLE (to June 2002); and the Meta-register of current controlled trials.

Study selection Due to the low incidence of BE a low yield of trials was expected so cohort and case-controlled studies were included where suitably matched control or comparison groups had been studied. The intervention was the administration of penicillin compared to no such administration before a dental procedure in people with an increased risk of BE. Outcomes of interest were: mortality or serious adverse event requiring hospital admission; development of endocarditis following any dental procedure in a defined time period; development of endocarditis due to other non-dental causes; any recorded adverse events to the antibiotics; and cost implications of the antibiotic provision for the care of those patients who develop endocarditis.

Data extraction and synthesis Two reviewers independently selected studies for inclusion, then assessed quality and extracted data from the included study.

Results One case-control study met the criteria. This study included participants who died because of the endocarditis (using proxys). It collected all the cases of endocarditis in the Netherlands over 2 years, finding 24 people who developed endocarditis within 180 days of an invasive dental procedure. Controls attended local cardiology outpatient clinics for similar cardiac problems, had undergone an invasive dental procedure within the past 180 days and were matched by age with the cases. No significant effect of penicillin prophylaxis on the incidence of endocarditis could be seen. No data were found on other outcomes.

Conclusions There is no evidence about whether penicillin prophylaxis is effective or ineffective against bacterial endocarditis in people at risk who are about to undergo an invasive dental procedure. There is a lack of evidence to support published guidelines in this area. It is not clear whether the potential harms and costs of penicillin administration outweigh any beneficial effect. Ethically practitioners need to discuss the potential benefits and harms of antibiotic prophylaxis with their patients before a decision is made about administration.

\section{Commentary}

This new systematic review looks at the evidence as to whether penicillin prophylaxis is of any benefit in the prevention of infective endocarditis. Not surprisingly the authors conclude that due to the lack of randomised clinical trials (RCT) on this topic, no conclusions can be drawn. The reasons for the lack of RCTs is based upon the relatively rare occurrence of infective endocarditis, lack of precise details as to who is at risk from this condition, and poor understanding as to what dental procedures to cover. With respect to the latter, some authorities would say 'those procedures which result in a significant bacteraemia'.

However, many routine oral hygiene procedures also result in a significant bacteraemia, yet no cover is provided for these measures.

This review does leave the dental practitioner and other dental care professionals in somewhat of a quandary. There is no evidence to support the efficacy of penicillin in preventing infective endocarditis. However, we still continue to administer these drugs to our patients if they fall into the "at risk" category. Since the benefits have not been established, the converse needs to be considered, notably the unwanted effects of these drugs. Life threatening anaphylaxis is the most significant adverse event that can arise from penicillin administration. Despite taking a careful medical history, some patients may be unaware of a sensitivity to this drug and therefore be at risk from an anaphylactic reaction. It has been estimated that patients who require antibiotic cover are 5 times more likely to die from a penicillin-induced anaphylaxis than from infective endocarditis. We are recommended to discuss the potential benefits and harms of antibiotic prophylaxis with our patients before deciding to proceed. The risks of penicillin seem greater than the benefits. However, the fear of litigation does cloud this whole issue and practitioners will be influenced by this threat.

The whole topic is confusing. By providing penicillin prophylaxis, we are attempting to prevent a serious, but rare disease. In so doing we may be putting our patients at greater risk. It is surprising that our clinical practice still continues amongst so much confusion and uncertainty.

\section{Robin Seymour}

School of Dental Sciences, University of Newcastle, Newcastle, UK

Evidence-Based Dentistry (2004) 5, 46.

doi:10.1038/sj.ebd.6400258 